\title{
Authentication and Payment in Future Mobile Systems
}

\author{
Günther Horn ${ }^{1} \quad$ Bart Preneel ${ }^{2}$ \\ ${ }^{1}$ Siemens AG, Corporate Technology, ZT IK 3, D-81730 München, Germany, \\ guenther.horn@mchp.siemens.de \\ ${ }^{2}$ Katholieke Universiteit Leuven, Dept. Electrical Engineering-ESAT, \\ Kardinaal Mercierlaan 94, B-3001 Heverlee, Belgium \\ bart.preneel@esat.kuleuven.ac.be
}

\begin{abstract}
This article presents an efficient public-key protocol for mutual authentication and key exchange designed for future mobile communications systems. The paper also demonstrates how a micropayment scheme can be integrated into the authentication protocol; this payment protocol allows for the provision of incontestable charging. The problem of establishing authenticated public keys through cross-certification is addressed.
\end{abstract}

Key words: Authentication, Public-key protocols, Mobile Systems, Micropayments, Crosscertification

\section{Introduction}

\subsection{The future of mobile systems}

Mobile communications is one of the fastest growing sectors of the IT industry. Second generation digital cellular systems are in widespread use and achieve a high penetration rate in many countries. The system with the greatest impact is GSM (Global System for Mobile Communications). In October 1999 there were over 300 GSM networks operating in 133 countries and serving 210 million subscribers. It is expected that between 700 million and one billion subscribers will be served by GSM networks by the year 2005 [19].

While current second generation systems will continue to play an important role, a new third generation system, the UMTS (Universal Mobile Telecommunications System) is shortly to be introduced. UMTS is one of the major new third generation mobile communications systems being developed within the framework defined by the ITU (International Telecommunications Union) [29], known as IMT-2000. UMTS needs to be capable of co-existing and working with existing second generation mobile communications technologies so that operators can choose to re-use their existing infrastructure assets and expertise. A phased introduction of UMTS hardware and services is expected with coverage, capability and number of operators growing over time. Commercial UMTS services are expected to be introduced in Europe by 2002 [43]. UMTS will provide a wider spectrum of services than today's systems, ranging from simple voice telephony to high speed, high quality multimedia services, regardless of physical location of the user, using radio frequency access to a convergent network of fixed, cellular and satellite components.

Though much attention is currently focused on the early years of deployment, UMTS is being defined with a view to the long term. In time it is expected that its capabilities will extend far beyond those envisaged today. 
UMTS has so far been standardised by ETSI (European Telecommunications Standards Institute) [14]. Starting in 1999 the technical specification work for UMTS has been taken over by a new body, the "Third Generation Partnership Project" known by the acronym "3GPP" [1]. The partners cooperating in 3GPP are the standards organisations ETSI, the Japanese ARIB and TTC, the Korean TTA, the Chinese CWTS and the North American Committee T1. The specification work will be based on the evolved GSM core networks and the radio access technologies that the partners support. Also the security specification work has moved from ETSI to 3GPP. Work is currently concentrated on UMTS release '99 security specifications to be completed by the end of 1999 .

\subsection{Mobile systems security}

As for second generation systems such as GSM (cellular) and DECT (cordless), the fundamental security requirement for UMTS is to ensure that the level of security is at least as high as that in existing wired telecommunications networks. The security specifications for GSM and DECT can be found in [15, 16] and [17] respectively. For an overview of GSM security, see Vedder [44]. The necessary security features to meet this fundamental requirement include: confidentiality on the air interface (which is much more vulnerable to eavesdropping than a wired interface), anonymity of the user and, most importantly, authentication of the user to the network in order to prevent fraudulent use of the system. While these features are already provided in existing second generation systems, they will be further developed and enhanced by the incorporation of additional features in UMTS.

UMTS release '99 security features will additionally include authentication of the network to the user, and integrity protection of selected signalling messages in order to prevent certain active man-in-the middle attacks. UMTS release '99 specifications are based on the premise that as much as possible of the GSM core network be reused. UTMS release '99 security will use secret key techniques, as in second generation systems.

This paper considers issues which are of relevance for later phases of UMTS whose specifications are to be released after 1999. For these phases, the number and variety of competing public and private network operators and service providers will grow larger. The resulting potential network complexity also suggests that new techniques of managing the cryptographic keys necessary for the provision of these security features may be required. This is a very natural setting for the application of public-key cryptographic techniques. The use of public-key techniques may offer additional benefits such as an improved protection of the confidentiality of the user's identity, and non-repudiation services. Although applied successfully in other areas, public-key cryptography has not previously been used in mobile communication environments due to performance constraints. It was not deemed suitable for second generation systems because of the resulting length of messages and the computational loads.

To overcome these problems, a new protocol was developed for mutual authentication and key exchange, run between a user and a UMTS network as part of the network access procedure; this protocol may also be used to provide mutual authentication and key exchange between a user and a value-added service provider in a UMTS network as part of a UMTS application. The latter case is considered in this paper. The protocol was designed to fit the performance constraints of mobile networks. It is described in section 2 below. Its design exploits the advances in two fields: crypto-controller smart cards (which have a co-processor which efficiently supports public-key cryptographic mechanisms) and elliptic-curve cryptosystems (which permit the use of smaller cryptographic parameters). The new protocol was successfully implemented and tested in the collaborative research project ASPeCT (Advanced Security for Personal Communications Technologies) which was funded by the European Union under the ACTS programme. An extended version of the protocol to include on-line TTPs (Trusted Third Parties) is presented in section 5. The choice of cryptographic algorithms and certificate formats is discussed in section 6 .

Another crucial issue in the security of mobile systems is that users may want to protect themselves against incorrect bills. Therefore it may be necessary to provide undeniable evidence that claims related to user charges are correct. Such undeniable evidence can be obtained if 
the user pays for the provision of services on-line using an electronic payment system which provides non-repudiation of crucial payment data. This approach may be applied to both basic telecommunication services (e.g. voice and data calls) and value-added services (e.g. location based information services). The ASPeCT project focussed on the latter as new forms of payment for these services are likely to be accepted by the market earlier. The ASPeCT project demonstrated the feasibility of a solution to the problem of securely billing for the provision of a value-added service using the aforementioned authentication protocol and a suitable micropayment scheme. The authentication protocol is used for initialisation of the micropayment system. The integration of the initialisation of payment with the authentication protocol is described in section 2 . The payment protocol itself is described in section 3. A re-initialisation of payment protocol is described in section 4 .

\section{Authentication and initialisation of payment protocol}

In the following, $U$ denotes the user, represented by his User Identity Module (a security module which in practice will be implemented on a smart card) and $V$ denotes the UMTS value added service provider, or, in short, VASP. The design principles for the protocol can be found in section 2.2 .

\section{$2.1 \quad$ Protocol goals}

The goals to be achieved by the end of a successful protocol run are the following (most definitions are from the Handbook of Applied Cryptography by Menezes, van Oorschot and Vanstone [36]; see also $[25,26])$ :

1. mutual entity authentication of $U$ and $V$; here (unilateral) entity authentication is defined as the prcoess whereby one party is assured (through acquisition of corroborative evidence) of the identity of a second party involved in a protocol, and that the second has actively participated [36, Def. 10.1];

2. agreement between $U$ and $V$ on a secret session key $K$;

3. mutual implicit key authentication; (implicit) key authentication is the property whereby one party is assured that no other party aside from a specifically identified second party may gain access to a particular secret key [36, Def. 12.6];

4. mutual key confirmation between $U$ and $V$; (unilateral) key confirmation is the property whereby one party is assured that a second (possibly unidentified) party actually has possession of a particular secret key [36, Def. 12.7];

5. mutual assurance of key freshness; a key is fresh (from the viewpoint of one party) if this party has assurance that it was generated during the current run of the key establishment protocol [36, Sect. 12.2.2];

6. mutual key control: this is the property whereby a key is derived from joint information, and neither party is able to control or predict the value of the key [36, Sect. 12.2.2].

7. non-repudiation of origin by $U$ for relevant data sent from $U$ to $V$; non-repudiation of origin is intended to protect against the originator's false denial of having created the content of a message and of having sent a message [26].

8. confidentiality of the user identity with respect to third parties;

9. exchange of certified public keys;

10. initialisation of the payment mechanism. 
Note that the combination of (implicit) key authentication and key confirmation is denoted explicit key authentication [36, Def. 12.8].

These goals are motivated by the following observations: a user $U$ must be authenticated by the network side $V$ before he may obtain service. Conversely, $U$ may desire to be assured of the identity of the service provider $V$ so that he can have trust in the service. Both parties need to agree on a session key for the purposes of encrypting or authenticating messages exchanged during the session. In order to communicate securely the parties need to be assured with whom they share the session key (goal 3). It could be argued that key confirmation was dispensable because no meaningful communication during the session would be possible if one party was not in possession of the correct session key; however, the property is useful because it allows to notice a failure already during the key agreement phase and then abort the protocol. Key freshness is important as it prevents the replay of old keys. Key control of the user may not be required if he trusts the network side to provide good session keys. However, key control is closely related to key freshness in so far as the mechanisms ensuring mutual key freshness in the protocol presented here also ensure mutual key control. The non-repudiation feature is motivated by the requirement for incontestable charging described in section 1.2. The confidentiality of the user identity is motivated by the vulnerability of the air interface to eavesdropping. The final feature allows for improved efficiency by integrating authentication and payment into a single protocol.

\subsection{Principles for the selection of security mechanisms}

One principle in the design of the protocol was to limit the computational effort on the user side at the expense of the network side, because it is assumed that the user will be represented by a smart card which has limited computational capabilities. Another principle was to allow for messages that are as short as possible. A way to arrive at shorter messages is the use of elliptic curve cryptosystems [35]. While the protocol does in no way mandate the use of these cryptosystems it is designed in such a way that their advantages can be best exploited. Another way to arrive at shorter messages is the use of a streamlined certificate format which provides certificates much shorter than X.509 certificates. (For more details see section 6.2.)

The choice of the security mechanisms was guided by the following considerations: nonrepudiation of data sent by the user requires a digital signature system on the user side. This signature system is then also used for authentication of the user for efficiency reasons. For session key establishment, a key agreement scheme (similar to the ElGamal scheme [13]) with implicit key authentication of the network was chosen because entity authentication of the network can then be obtained for little extra cost. (See discussion in section 2.5 below.) The protocols were chosen in such a way that their description is independent of the choice of the signature systems used by the user and the certification authority, respectively. They are also independent of the choice of the finite group in which the exponentiations required in the key agreement scheme are computed.

\subsection{Prerequisites}

\subsubsection{Cryptographic functions}

It is assumed that the following cryptographic functions can be executed by any participant:

- A symmetric encryption function where $\{M\}_{K}$ denotes the encryption of message $M$ with key $K$. It is assumed that the cryptographic algorithm is resistant against known cryptanalytic attacks such as code-book attacks and chosen plaintext attacks.

- A random (or pseudo-random) number generator.

- Functions $h 1, h 2$ and $h 3$ which are specified in section 2.3 .3 below.

- Multiplications in a finite group $G$ with generator $g$, (e.g., the multiplicative group of a finite field or a subgroup of an elliptic curve), in which the Discrete Logarithm Problem is hard. 


\subsubsection{Further prerequisites}

- The identity $i d V$ of $V$ is assumed to be known to $U$ at the start of the protocol.

- $V$ has long-term secret and public key agreement keys $v$ and $g^{v}$ respectively, where $g$ is as above.

- $U$ possesses an asymmetric signature system with secret signature transformation $\operatorname{Sig}_{U}$. In case of a signature with appendix, $\operatorname{Sig}_{U}()$ denotes only the appendix. Digital signatures with appendix are defined in ISO/IEC 14888 [27]; these are digital signatures for which (part of) the signed data cannot be recovered from the data, and thus the signature is appended to the data.

- There is a valid certificate cert $U$ (issued by a certification authority $C A U$ with identity $i d C A U)$ on the public key of the asymmetric signature system of $U$, available at $U$.

- There is a valid certificate $\operatorname{cert} V$ (issued by a certification authority $C A V$ with identity $i d C A V$ ) on the public key agreement key $g^{v}$ of $V$, available at $V$.

- $U$ possesses the public key necessary to verify certificates issued by $C A V$.

- $V$ possesses the public key necessary to verify certificates issued by $C A U$.

Note. In a practical situation, the last two prerequisites may not be satisfied. In this case certificate chains may have to be used, see also section 2.5.2, item 7 and section 5.2.

\subsubsection{Functions $h 1, h 2, h 3$}

Here, the requirements on the functions $h 1, h 2$ and $h 3$ are listed explicitly. The following definitions are useful here: definitions 1, 2 and 3 are well-known [36, p. 323ff.], while definitions 4 and 5 are weak forms of the MAC-property and of pseudo-randomness which we believe are sufficient in our context. Concatenation is indicated by $\|$.

1. A function $h$ is preimage resistant (one-way), if for essentially all outputs $y=h(x)$ it is computationally infeasible to find any input $x^{\prime}$ with $y=h\left(x^{\prime}\right)$ ( $x^{\prime}$ may or may not be equal to $x)$.

2. A function $h$ is partial-preimage resistant (locally one-way), if for essentially all outputs, if part of the input is known it is still hard to find the remainder, i.e. it is not easier than brute-force.

3. A function $h$ is collision resistant (strong collision resistant), if it is computationally infeasible to find two inputs $x^{\prime} \neq x$ which are mapped to the same value $y=h(x)=h\left(x^{\prime}\right)$.

4. A function $h$ is weakly computation resistant (weak MAC-property) if it is computationally infeasible to find a pair $(x, h(K \| x))$ without knowing $K$, provided that no other pair $\left(x^{\prime}, h\left(K \| x^{\prime}\right)\right)$ is known (here the value of $x$ can be chosen by the opponent).

5. A function $h$ is a weakly pseudo-random function if, for secret random key $K$ not used before and for known random $x$, the output $h(K \| x)$ is indistinguishable from a random output.

Note on the Definitions. There are some dependencies between these properties, e.g., a partial-preimage resistant function is preimage resistant. Note also that definition 4 is indeed much weaker than the usual MAC-property (cf. [36, p. 325]) because it is assumed for the weak MAC-property that an attacker does not even have one valid (message, MAC) pair against which he could test attempted forgeries. Definition 4 is not strictly weaker than definition 5 since definition 4 allows the opponent to choose the value of $x$. 
Definition 5 can be made operational in the following way: let $h$ be a publicly known function. Given parties Alice and Eve, Alice chooses random parameters $K_{i}, x_{i}, R_{i}(i=1,2, \ldots)$ and computes $H_{i}:=h\left(K_{i} \| x_{i}\right)$. Alice makes $x_{i}, R_{i}$ and $H_{i}$ known to Eve and keeps $K_{i}$ secret. Eve tries to break the function $h$ by guessing which of the two parameters $H_{i}$ and $R_{i}$ is computed from $K_{i}$ and $x_{i}$ by applying $h$. If Eve has only about a $50 \%$ chance to make the correct guess then $h$ fulfils definition 5. Note that this does not imply that such a function $h$ (in practice a hash function) can be used to define a pseudo-random function because the opponent has no control over the input $x_{i}$ and can only observe a single input-output pair $\left(x_{i}, H_{i}\right)$ for each choice of $K_{i}$. Nothing is implied by definition 5 about repeated applications of $h$ with the same value for $K_{i}$.

It is required that the functions $h 1, h 2$ and $h 3$ are hash functions (i.e. they map inputs of arbitrary finite lengths to fixed length outputs) which are easy to compute and that

1. $h 1$ is a partial-preimage resistant, weakly computation resistant and weakly pseudo-random function.

2. $h 2$ is a partial-preimage resistant, weakly computation resistant function.

3. $h 3$ is a collision resistant function.

There are a number of practical cryptographic functions that are assumed to be at least collision resistant, e.g., SHA-1 [18, 24], RIPEMD-160 [12, 24] and ISO/IEC 10118-2 [24]. It is common practice in cryptographic applications to assume that these hash functions are also preimage and partial-preimage resistant, though this issue needs further investigation. Due to [36, p. 331] hash functions should not be used as pseudo-random functions unless the randomness requirements are "clearly understood". This does not contradict our assumption that a practical hash function fulfils our definition 5, (see corresponding note following definition 5).

Let $h$ be one of these well-known hash functions. Then we claim that the functions

1. $h 1(x):=h(x)$,

2. $h 2(x):=\operatorname{trunc}(n, h(x))$ where $\operatorname{trunc}(n, y)$ returns the $n$ least significant bits of $y$ ( $n$ being significantly shorter than the length of the hash value, but long enough to prevent successful guessing),

3. $h 3(x):=h(x)$,

satisfy our requirements.

\subsection{Protocol description}

USER $U$

$\stackrel{g^{s} \| i d C A V}{r\|h 2(K\|r\| i d V)\| \text { ch_data }\|T V\| \operatorname{cert} V}$
$\stackrel{\left.\operatorname{Sig}_{U}\left(h 3\left(g^{s}\left\|g^{v}\right\| r\|i d V\| \text { ch_data }\|T V\| \alpha_{T} \| I V\right)\right)\|\operatorname{cert} U\| \alpha_{T} \| I V\right\}_{K}}{\stackrel{\text { VA }}{\longleftrightarrow}}$

Figure 1: The authentication and initialisation of payment protocol.

At the start of the protocol (see Figure 1), $U$ generates a random number $s$ and then computes $g^{s}$ which he sends to $V$, together with the identity $i d C A V$ (see remark on certificate verification keys in section 2.5). 
On receipt of the first message, $V$ does not know with whom he is communicating. $V$ generates a random number $r$, computes $\left(g^{s}\right)^{v}$ and then a session key $K:=h 1\left(\left(g^{s}\right)^{v} \| r\right)$. He demonstrates knowledge of the session key $K$ by computing the hash value $h 2(K\|r\| i d V)$ which he then sends to $U$, together with $r$, his certificate cert $V$ and additional data needed as input to the payment scheme (a timestamp $T V$ and charging-relevant data ch_data).

On receipt of the second message, $U$ computes the key $K=h 1\left(\left(g^{v}\right)^{s} \| r\right)$. He then checks the hash value $h 2(K\|r\| i d V)$ and he thus knows that $V$ actually has the session key $K$. $U$ generates random numbers $I V$ and $\alpha_{0}$, computes $\alpha_{T}=F_{I V}^{T}\left(\alpha_{0}\right)$ and signs the hash value of the concatenation $g^{s}\left\|g^{v}\right\| r\|i d V\|$ ch_data $\|T V\| \alpha_{T} \| I V$. Here, $F_{I V}, T, \alpha_{0}, \alpha_{T}$ and $I V$ have significance only for the payment scheme, not for the protocol goals stated above, and are therefore explained in section 3. $U$ concatenates the signed hash with his certificate cert $U$ and with $\alpha_{T}$ and $I V$. $U$ then encrypts the concatenated parameters with $K$.

On receipt of the third message, $V$ first deciphers the message elements using $K$. He retrieves the certificate cert $U$ and, after verification of the certificate, he verifies $U$ 's signature. $V$ stores the signature and the corresponding message for later use in the payment scheme (see section 3 below).

\subsection{Discussion}

\subsubsection{Meeting the protocol goals}

It is shown in this section that the goals listed in section 2.1 are met.

1. Implicit key authentication and key confirmation of $V$ : implicit key authentication of $V$ is provided because $V$ can derive the key $K$ only by knowing the secret key agreement key $v$ which belongs to $V$ as certified by cert $V$. The inclusion of $h 2(K\|r\| i d V)$ in the second message gives key confirmation from $V$ to $U$. Note that explicit key confirmation demands that the session key is used in the key distribution itself. Some protocols do not use the session key in the key distribution protocol itself in order to avoid leaking information on the session key. Such information leakage on the session key is minimised in our protocol by the inclusion of random numbers in the encrypted messages and by the assumptions on the symmetric encryption function. This shows one direction of the goals 3 and 4 .

2. Key freshness: the key $K$ is constructed using the random number $r$ generated by $V$. This, together with the use of the random number $s$ generated by $U$, guarantees key freshness to both sides. This shows goal 5 .

3. Entity authentication of $V$ : by key confirmation, $U$ is assured that a second party actually has possession of the session key $K$. By implicit key authentication of $V, U$ is assured that this second party is $V$. By key freshness, evidence of the possession of the secret key must have been produced during the current protocol run, i.e., $V$ has actually participated. This provides entity authentication of $V$ according to the definition in section 2.1. (For the argument presented here see also Rueppel and van Oorschot [42].) This shows one direction of goal 1.

4. Key confirmation and authentication of $U$ : the encryption of the certificate $\operatorname{cert} U$ with $K$ in the third message provides key confirmation. The inclusion of $g^{s}\left\|g^{v}\right\| r$ in the signed part of the third message provides implicit key authentication from $U$ to $V$ as it confirms the origin of $g^{s}$ and links this value to $g^{v}$ and $r$. The inclusion of the random number $r$ generated by $V$ in the signed part of the third message provides entity authentication of $U$. This shows the other directions of goals 1,3 and 4 .

5. Session key agreement: it is clear from the above that goal 2 is met.

6. Joint key control: both parties contribute to the value of the key by controlling the parameters $s$ and $r$ respectively. However, see section 2.5.2, item 5. This shows goal 6 . 
7. Non-repudiation: $U$ 's signature provides non-repudiation of the signed data. Note also that in order to achieve non-repudiation it has to be ensured by means outside this protocol that the certificate cert $U$ of $U$ is still valid. Furthermore, in order to achieve non-repudiation, it may be required in addition that $V$ submits $\operatorname{Sig}_{U}(\ldots)$ to a trusted time-keeper who signs $\operatorname{Sig}_{U}(\ldots)$ together with a timestamp and returns it to $V$. Otherwise, $U$ could repudiate a signature claiming that the signature was generated by an impostor after the certificate had been revoked. Whether this additional measure should be implemented, however, depends on the security policy and on a trade-off between a higher security level and additional effort. This shows goal 7.

8. Confidentiality of user identity: this is ensured by encrypting the user's certificate and the signature (see section 2.5.2, item 1) with the symmetric encryption function using $K$. This shows goal 8.

9. Exchange of certified public keys: this follows immediately from the fact that $V$ sends his certificate $\operatorname{cert} V$ to $U$ in step 2, and $U$ sends his certificate $\operatorname{cert} U$ to $V$ in step 3. This shows goal 9 .

10. Initialisation of the payment mechanism: the user signs a commitment $i d V \|$ ch_data $\|$ $T V\left\|\alpha_{T}\right\| I V$ in the course of the protocol. For the meaning of this commitment in the context of the payment system see section 3 . This shows goal 10 .

\subsubsection{Protocol features and general attacks}

1. Encryption of the signature: the signature is encrypted for two reasons. Firstly, an attacker may be able to detect the identity of $U$ by verifying the signature. This might be possible in a scenario where the attacker has access to the public keys of the users and he assumes that the originator of the signature is one of a small group of users (here 'small' depends on the time needed to verify a signature). Secondly, an attacker may carry out an entity spoofing attack: if the signature was not encrypted then the attacker could replace the user's certificate with his own certificate and the user's signature with his own signature, as all signed data would then be public. $V$ would then be lead to believe that he shares the key $K$ with the attacker, while in fact he shares $K$ with the user. Encryption of the payment related parameters $\alpha_{T}$ and $I V$ is not strictly necessary, but these parameters may be encrypted with the rest of the message for convenience of implementation.

2. Inclusion of $i d V$ in the second message: this prevents so-called source-substitution attacks (as described in [36, remark 12.54]). These can also be avoided by making sure that the certification authority checks that the user is in possession of the corresponding private key before it issues a certificate on the public key. The inclusion of $i d V$ is simpler and prevents the need to detail the duties of a certification authority in this context.

3. Inclusion of $\boldsymbol{i d V}$ in the third message: $i d V$ must be included in order to indicate the intended recipient of the signature. This is related to the use of the signature in the payment scheme: the signature, together with the payment tokens sent in the payment protocol (see section 3 below), serves as a proof of payment. If $i d V$ would be omitted, anyone who intercepts the signature and the tokens and presents them to the broker of the payment scheme could collect money fraudulently.

4. Inclusion of $\boldsymbol{r}$ in the hashed part of the second message: this strengthens the protocol against time-memory trade-off attacks (see Hellman [21] and Borst et al. [6]) by precluding efficient precomputations of $h 2()$. Note that time-memory trade-off attacks are not feasible if the key space is large enough.

5. Imbalance in joint key control: $V$ has somewhat more control over the key $K$ than $U$. On receipt of $g^{s}, V$ can compute $K$ for $2^{q}$ values of $r$ before responding and, in this way, 
control approximately $q$ bits of $K$, as pointed out by Mitchell et al. [37]. However, $q$ is severely limited by the computational capabilities of $V$ and the time available to respond to the first message. Therefore, the imbalance in key control is not considered a problem for this protocol in practice.

6. Multiple protocols and key separation: Suppose that as part of a run of a second, different protocol subsequent to a run of the authentication protocol presented in this section, an attacker sends data to $U$ that $U$ encrypts with the same session key $K$ and returns to the attacker. It is then possible that the attacker could exploit the lack of key separation and replace the third message of the authentication protocol with another message chosen by the attacker and encrypted by $U$ in the second protocol. Whether such an attack is feasible depends on the context in which the authentication protocol is used. If the attack is a potential source of concern, then in any subsequent protocol $K$ should be replaced by a key derived from $K$. One solution could be to use a variant of the function $h 1$.

7. Certificate verification keys: since $U$ has limited space for storing public keys, $U$ will not be able to verify certificates issued by an arbitrary certification authority. Therefore, in the first message, $U$ tells $V$ the identity idCAV of a certification authority $C A V$ (certification authorities $C A V 1, C A V 2, \ldots$ ) whose certificates he is able to verify. We assume that $V$ has got a certificate issued by this authority (one of these authorities). If this is not the case then an extended version of the protocol has to be run to obtain such a certificate (see section 5.1).

8. Identification of multiple users: when the protocol is run concurrently by many users it is necessary to identify which message belongs to which user. This problem is assumed to be taken care of by the underlying communication system and, hence, need not be addressed by the security protocol. Temporary channel identifiers can be used to provide user anonymity, see for example Vedder [44].

9. Perfect forward secrecy: a protocol is said to provide perfect forward secrecy if the compromise of long-term keys does not compromise past session keys [36, Def. 12.16]. The protocol does not provide perfect forward secrecy according to this definition. However, it provides unilateral perfect forward secrecy: if the user's long-term secret is disclosed then still nothing can be inferred about previously established session keys. But the same is, of course, not true if $V$ 's long-term secret, the key agreement key $v$, is disclosed.

10. Known-key attacks: a protocol is said to be vulnerable to a known-key attack if compromise of past session keys allows either a passive adversary to compromise future session keys, or impersonation by an active adversary in the future [36, Def. 12.17]. Because the session key is generated using a partial pre-image resistant and (weakly) pseudo-random function $h 1$, nothing can be derived from previous session keys about the input with which they were generated and, consequently, about secrets derived in future protocol runs.

\subsubsection{Possible attacks and the choice of the properties of $h 1, h 2$ and $h 3$}

The weak pseudo-randomness property makes $h 1$ suitable for key derivation in our context. Note that only one session key $K$ is derived from the master key $g^{s v}$.

Furthermore, the proposed protocol avoids the following attacks: an attacker Eve may try to forge the second message in three ways by attacking the function $h 1$ or the function $h 2$ or the concatenated function $H$ (see 3 . below):

1. Eve may try to find a valid pair $(r, K)=\left(r, h 1\left(g^{s v} \| r\right)\right)$. If she could do that she could compute $h 2(K\|r\| i d V)$ from this. This is impossible by the weak MAC-property of $h 1$ because she does not know $g^{s v}$.

2. Eve may try to find a valid pair $\left(r^{\prime}, h 2\left(K\left\|r^{\prime}\right\| i d V\right)\right)$, possibly after having seen the valid pair $(r, h 2(K\|r\| i d V))$ generated by $V$. But this would not help Eve because when $U$ tries 
to verify the forged second message $\left(r^{\prime}, h 2\left(K\left\|r^{\prime}\right\| i d V\right)\right), U$ computes $K^{\prime}=h 1\left(g^{s v} \| r^{\prime}\right)$ which by the weak pseudo-randomness of $h 1$ is almost certainly different from $K$ for $r \neq r^{\prime}$. U's verification would be successful only if $h 2\left(K^{\prime}\left\|r^{\prime}\right\| i d V\right)=h 2\left(K\left\|r^{\prime}\right\| i d V\right)$, but then Eve would have generated a valid pair $\left(r^{\prime}, h 2\left(K^{\prime}\left\|r^{\prime}\right\| i d V\right)\right)$ without knowing $K^{\prime}$ and without having seen another valid pair involving $K^{\prime}$, which contradicts the weak MAC-property of $h 2$.

3. Eve may attack the concatenated function $H(L, r):=h 2(h 1(L \| r)\|r\| i d V)$ directly and may try to find a valid pair $\left(r^{\prime}, H\left(g^{s v}, r^{\prime}\right)\right)$, possibly after having seen the valid pair $\left(r, H\left(g^{s v}, r\right)\right)$, generated by $V$. However, Eve would then also have generated a valid pair $\left(r^{\prime}, h 2\left(K^{\prime}\left\|r^{\prime}\right\| i d V\right)\right)$ where $K^{\prime}=h 1\left(g^{s v} \| r^{\prime}\right)$ and $K^{\prime} \neq K$ almost certainly by the weak pseudo-randomness of $h 1$, hence this contradicts the weak MAC-property of $h 2$.

\section{The payment protocol}

\subsection{General}

There is a recognised need for so-called micropayment systems, that are suitable for the efficient payment of small, frequently recurring, variable amounts. Applications range from electronic publishing to metering, telecommunications and information services and video-on-demand. A series of payments should be made to the same vendor over a period of time so that the vendor can aggregate the individual payments and spread the cost for clearing them with the broker over a larger number of payments.

The micropayment system presented here is based on Pedersen's tick payment protocol. For a detailed discussion, the reader is referred to [32, 40], however its main features are summarised below. The novelty is not the payment protocol itself, but the way in which it is integrated with the authentication protocol proposed for the mobile system UMTS (cf. section 1) and the payment scenario for basic and value added services in UMTS.

The cryptographic mechanism employed in the tick payment protocol is based on Lamport's password scheme [33]. Later Pedersen, Rivest and Shamir [41] and Anderson et al. [2] independently proposed the same mechanism for the payment of small amounts. Hauser et al. [20], who were aware of Pedersen's work, proposed to integrate the mechanism with IBM's iKP (Internet Keyed Payment Systems) protocol for credit-card based electronic transactions. Jutla and Yung have extended this approach [31].

Figure 2 provides a simple model of the payment system, and introduces the three parties: payer, payee and broker. The proposed payment transaction has two phases: an initialisation phase in which the payer (in our scenario the user of a value added UMTS service) commits to initial values of the payment scheme by a digital signature, and an actual payment phase in which payments are made to the payee (in our scenario a UMTS value added service provider or VASP) by successively releasing preimages of an initial value $\alpha_{T}$ under a one-way function $F$. Initialisation is performed as part of the authentication protocol described in the previous section. The meaning of the parameters signed in the third message of that protocol and the detailed working of the tick payment protocol performed in the payment phase are described in the following.

\subsection{Goals}

A payment system has to satisfy a large number of goals; one distinguishes between privacy requirements and integrity requirements for the three parties (payer, payee, and broker). This paper focuses on the actual payment transaction between payer and payee, and gives less attention to the other aspects of a payment protocol (withdrawal and deposit); the full model and detailed requirements of the payment system are outside the scope of this paper. The privacy aspects of the payment transaction are addressed by user identity confidentiality and encryption (cf. section 2). The goals of the payment transaction with respect to integrity are described below. 


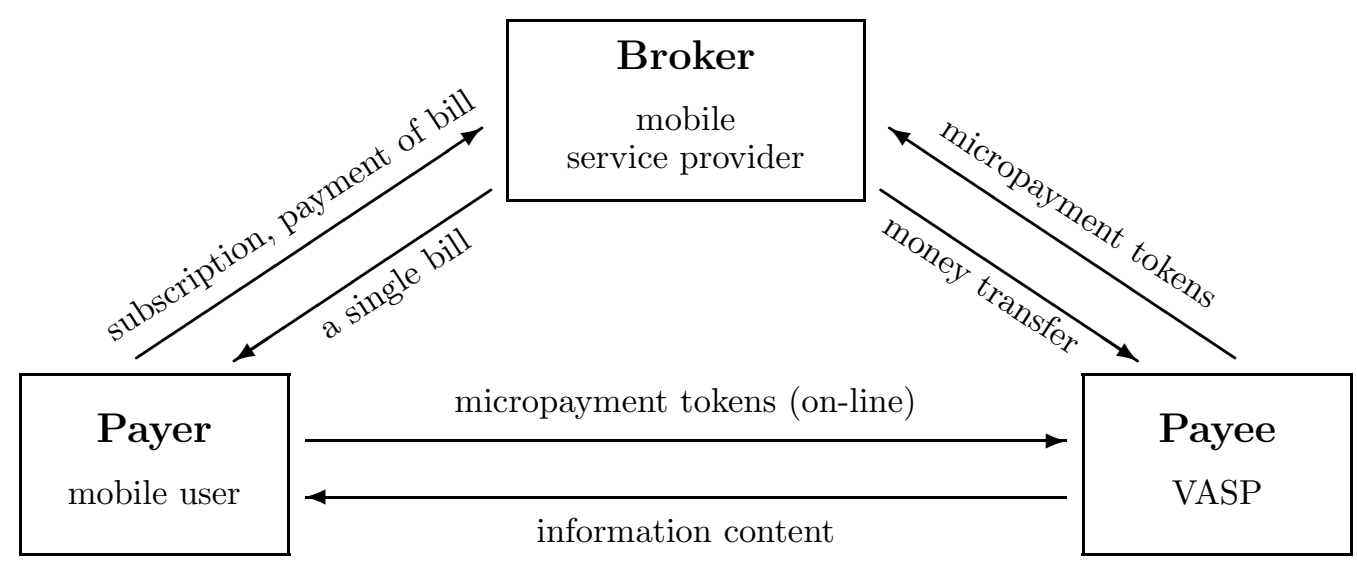

Figure 2: Model of the payment system.

\subsubsection{From the payer's point of view}

1. no one but the payer himself is able to pay using the funds of the payer;

2. the amount of the payment is exactly what the payer has specified;

3. only the payee specified by the payer can receive the payment.

\subsubsection{From the payee's point of view}

4. a payee can verify the amount, the payer and payee of a payment;

5. the payer cannot deny having made a verified (electronic) payment, i.e., he cannot later on stop or re-claim the corresponding (real world) payment (incontestable charging);

6. the payee can be certain of being credited for verified payments by the broker.

\subsubsection{From the broker's point of view}

7. the broker can verify the amount, the payer and payee of each payment;

8. the broker can detect double deposits of payments.

\subsection{Prerequisites}

The tick payment protocol has the following prerequisites:

- There is a public system parameter $T$ which gives the maximum number of ticks (the currency unit of the payment system, reminiscent of phone ticks) to which the user can commit himself by one signature.

- There is a public family $F$ of length-preserving one-way functions $F_{I V}:\{0,1\}^{n} \rightarrow\{0,1\}^{n}$, where $n$ is a public system parameter and $I V$ is an initialisation vector. (To be more precise, the functions $F_{I V}$ need to be one-way on $T$-th iterates, cf. [32, 40].)

Both $F$ and $T$ have to be chosen with care in order to avoid certain attacks (see discussion in section 3.5). 


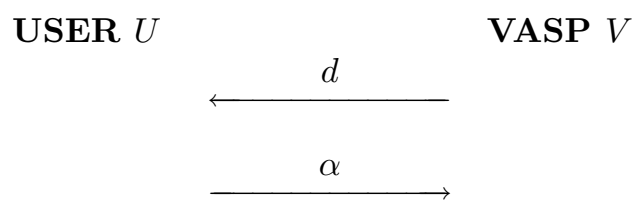

Figure 3: The tick payment protocol.

\subsection{Tick payment protocol description}

In the authentication and initialisation of payment protocol, the user commits to the parameters $i d V$, ch_data, $T V, \alpha_{T}$ and $I V$. Parameter $i d V$ is the identity of the payee (the VASP $V$ ), ch_data gives the conditions under which the payment is made (such as applicable tariff etc.), $T V$ is a timestamp generated by $V$, giving date and time of day, $I V$ is an initialisation vector by which the user selects a particular function $F_{I V}$ from the family $F$ of one-way functions, $\alpha_{0}$ is a random number selected by the user and $\alpha_{T}=F_{I V}^{T}\left(\alpha_{0}\right)$ is the initial value for the tick payments. All payments made are multiples of one "tick".

Whenever $V$ requests a payment from $U$, he sends a message to $U$ with the requested number $d$ of unit payments (ticks) to be made. It is assumed that $d \leq T$. If $d>T$ the amount has to be broken down accordingly. $U$ responds by releasing preimages of $\alpha_{T}=F_{I V}^{T}\left(\alpha_{0}\right)$ under the function $F_{I V}$. For the payment of the first $d 1$ ticks, the user sends the payment token $\alpha_{T-d 1}=F_{I V}^{T-d 1}\left(\alpha_{0}\right)$. On receipt, $V$ checks if $F_{I V}^{d 1}\left(\alpha_{T-d 1}\right)=\alpha_{T}$. For the payment of the next $d 2$ ticks, the user sends the payment token $\alpha_{T-d 1-d 2}=F_{I V}^{T-d 1-d 2}\left(\alpha_{0}\right), V$ checks if $F_{I V}^{d 2}\left(\alpha_{T-d 1-d 2}\right)=\alpha_{T-d 1}$, and so on. Only the last value received from the user has to be stored by $V$, together with the signature in the third message of the authentication and initialisation of payment protocol.

If the total number of requested ticks exceeds the maximum amount $T$ to which the user can commit himself by one signature, then either the authentication and initialisation of payment protocol has to be run again or, preferably, a simplified re-initialisation protocol is run which has only two messages and does not repeat mutual authentication. This re-initialisation protocol is presented in section 4.

$V$ can aggregate the payments from a single user, and clear the aggregated payments with the broker of the payment system, by presenting the signature by which the user committed to the initial values of the payment process and the last tick payment $\alpha_{T-d}$ made by the user. The VASP $V$ is then credited $d$ ticks by the broker.

\subsection{Discussion}

\subsubsection{Achievement of goals}

1. No one but the payer himself is able to pay using the funds of the payer. The reason is that the payer commits to the starting value $\alpha_{T}$ by his signature which identifies him uniquely. Therefore he is the only one who knows the preimages of $\alpha_{T}$ under the one-way function $F_{I V}$, provided the one-way function is appropriately selected. For the same reason, the payer cannot deny having made a verified (electronic) payment and try to stop or re-claim the corresponding (real world) payment.

2. The amount of the payment is exactly what the payer has specified: again, this depends on the appropriate choice of the one-way function, see the discussion by Pedersen [40].

3. The identity $i d V$ of the payee is included in the user's signature, therefore only the payee specified by the payer can receive the payment.

4. The payee can verify the payer and payee by checking the signature; he can verify the amount by checking that the $d$-th iterate of the one-way function $F_{I V}$ applied to the payment token 
received last equals the payment token received before (the starting value $\alpha_{T}$ respectively), where $d$ is the amount due.

5. The payee can be certain of being credited for verified payments by the broker because payee and broker, as well as any arbiter, can verify payments in the same way (see previous item). This assumes of course of that an agreement exists that the broker honours verifiable payments.

6. Check for double submission to broker: the signed commitment (that is, the signature in the third message of the protocol in Figure 1) contains $i d V$ and $r$, hence it is different for each session and payee. The broker checks for double submission of the same payment by comparing $i d V$ and the nonce $r$ in the signed commitment with previous commitments within a period determined by $T V$. If there is a match the broker refuses to honour the payment. No checking for double spending is required of the payee $V$. (Note that the broker could also perform the check based on $T V$ instead of on $r$, provided that the granularity of $T V$ is fine enough.) $T V$ has to be checked anyway when the re-initialisation protocol of section 4 is used.

\subsubsection{Further issues}

This section discusses some requirements of the function $F_{I V}$ and the choice of the related parameters $\left(\alpha_{T}\right.$ and $\left.T\right)$.

1. Re-use of starting value $\boldsymbol{\alpha}_{T}$ : it is in the interest of the payer not to re-use the starting value $\alpha_{T}$ in subsequent sessions, either with the same payee or with a different payee. If the starting value $\alpha_{T}$ is re-used, the payer may loose money in the following scenario. Assume that he pays for a total of $d 1$ ticks in the first session and a total of $d 2$ ticks in the second. The (fraudulent) payees could then for both instances claim $\max (d 1, d 2)$ from the broker (provided they could intercept the communication). Such a fraud could not be detected by the broker. The implication is that the payer has to generate $\alpha_{T}$ in a way that guarantees that it does not repeat (for example, by encryption of a counter with a sufficient period); if that is not possible, $\alpha_{T}$ should be chosen uniformly at random from a sufficiently large space, taking into account the birthday paradox (a 64 -bit random value $\alpha_{T}$ will repeat after approximately $2^{32}$ values).

2. Re-use of initialisation vector: the purpose of using a family of one-way functions instead of just one function is to make it much harder for a fraudulent payee to invert the one-way function by brute force with the help of pre-computations (see $[6,21])$. Therefore, a user should avoid re-using $I V$.

3. Collision resistance: this is not necessary for the function $F_{I V}$. It does not help a fraudulent payee if he is able to generate collisions for $F_{I V}$ because the starting value $\alpha_{T}$ is given to him by the payer.

4. The maximum amount: $T$ cannot be arbitrarily large. Rather, $F$ and $T$ must be chosen such that the probability that $F_{I V}{ }^{T}\left(\alpha_{0}\right)$ lies on a cycle is small for all $I V$. If $F_{I V}{ }^{j}\left(\alpha_{0}\right)$ lies on a cycle with length $c$ and $j+c<T$, then payments for $T-j$ and $T-j-c$ ticks cannot be distinguished as $F_{I V}{ }^{j}\left(\alpha_{0}\right)=F_{I V}{ }^{j+c}\left(\alpha_{0}\right)$. Bounds for $T$ as well as other results on the tick payment scheme are presented by Knudsen et al. [32].

\section{Re-initialisation protocol}

The re-initialisation protocol is introduced to increase the efficiency of the payment system. It is used in the following case: payment requests by the VASP have to be broken down in such a way that each individual request does not exceed the number of ticks left to the user. If the total 
number of ticks to be paid in a session is larger than the maximum amount $T$, then the user will eventually have spent all the ticks that he committed to in the initialisation phase. Consequently, a commitment for a new series of tick payments has to be provided. One solution would be to run the protocol of Figure 1 again, but a more efficient solution is to use the re-initialisation protocol depicted in Figure 4.

USER $U$ VASP $V$ ch_data $\| T V$

$\underline{\left\{\operatorname{Sig}_{U}\left(h 3\left(r\|i d V\| \text { ch_data }\|T V\| \alpha_{T} \| I V\right)\right)\left\|\alpha_{T}\right\| I V\right\}_{K}}$

Figure 4: The re-initialisation protocol.

The re-initialisation protocol is described as follows: the VASP sends charging-relevant data ch_data and a timestamp $T V$. The user generates new random numbers $I V$ and $\alpha_{0}$, computes $\alpha_{T}=F_{I V}^{T}\left(\alpha_{0}\right)$ and signs the hash value of the concatenation $r\|i d V\|$ ch_data $\|T V\| \alpha_{T} \| I V$. The meaning of $F_{I V}, T, \alpha_{0}, \alpha_{T}$ and $I V$ is explained in section 3. The parameter $r$ is identical to the challenge sent by the VASP and signed by the user in the run of the authentication and initialisation of payment protocol by which the current session was set up (cf. section 2). The user concatenates the signed hash with $\alpha_{T}$ and $I V$. The user then encrypts the concatenated parameters with $K$.

Note the similarity of the second message of the re-initialisation protocol in Figure 4 with the third message in the authentication and initialisation of payment protocol in Figure 1.

On receipt of the second message, the VASP retrieves the parameter $r$ pertaining to the current session with the user. The VASP decrypts the message with $K$ and verifies the signature. The VASP stores the decrypted message for later submission to the broker for clearance. In order to check the correctness of the payment data submitted for clearance by the VASP, the broker verifies the signature. He also checks for double spending by comparing $r$ and $T V$ with the stored values of $r$ and $T V$ relating to previously cleared payments.

The inclusion of $r$ binds the new commitment to the current session. The inclusion of the timestamp $T V$ in the signature prevents a re-play of a previous commitment within a session. The encryption of the last message with the session key $K$ agreed in the authentication and initialisation of payment protocol prevents the compromise of the identity of the user by a signer verification attack (as described in section 2.5.2 item 1).

Note. The encryption of the last message with $K$, of course, also binds the second message to the session. The broker, however, is only given the unencrypted commitment in the clearance of payment as it is undesirable that $K$ is stored over a possibly extended period and then disclosed to the broker. Therefore, the inclusion of $r$ is necessary.

\section{TTP related issues}

The protocol in section 2 assumes that the user and the VASP possess the public keys necessary to verify each other's certificates, or that they possess an authenticated copy of each other's public keys. However, if this is not the case, the parties might have to contact an on-line trusted third party (TTP) during the protocol in order to obtain a cross-certificate. Moreover, contacting an on-line TTP also allows for checking whether the certificate of the other party has been revoked. First the protocol with on-line TTP is described, and then cross-certificates are discussed. 


\begin{tabular}{|c|c|}
\hline \multicolumn{2}{|l|}{$g^{s}\|i d T T P\|\{i d U\}_{L}$} \\
\hline & $g^{s}\left\|\{i d U\}_{L}\right\| \operatorname{cert} V$ \\
\hline & $T T \|$ CertChain $(U, V)$ \\
\hline & $\stackrel{\| \operatorname{CertChain}(V, U)\}_{L} \| \operatorname{CertChain}(V, T)}{\longleftarrow}$ \\
\hline & $\overleftarrow{\|\left\{\operatorname{Sig}_{T}\left(h 3\left(g^{s}\|\operatorname{cid} U\| \operatorname{cid} V \| T T\right)\right)\right\}_{L}}$ \\
\hline \multicolumn{2}{|l|}{$r\|h 2(K\|r\| i d V)\|$ ch_data $\|T T\| \operatorname{CertChain}(U, V)$} \\
\hline \multicolumn{2}{|l|}{$\|\left\{\operatorname{Sig}_{T}\left(h 3\left(g^{s}\|\operatorname{cid} U\| \operatorname{cid} V \| T T\right)\right)\right\}_{L}$} \\
\hline$\left\{\stackrel{\operatorname{Sig}_{U}\left(h 3\left(g^{s}\left\|g^{v}\right\| r\|i d V\| \text { ch_data }\|T T\| \alpha_{T} \| I V\right)\right)}{\longrightarrow}\right.$ & \\
\hline$\left.\left\|\alpha_{T}\right\| I V \| L\right\}_{K}$ & \\
\hline
\end{tabular}

Figure 5: The authentication and key agreement protocol with on-line TTP.

\subsection{Protocol with on-line TTP}

The protocol in Figure 5 differs from the protocol in Figure 2 in the following aspects: $U$ also sends to $V$ the identity $i d T T P$ of his TTP, together with his own identity $i d U$ encrypted with the key $L:=g^{s w}$ where $g^{w}$ is the public key agreement key of the TTP. On receipt of the first message, $V$ contacts the TTP of the user and forwards the information sent by the user together with his certificate cert $V$. It is assumed that the user's identity is sufficient for the TTP to retrieve the appropriate certificate. The TTP decrypts $\{i d U\}_{L}$ and verifies whether the certificate of $U$ has been revoked; it might also perform the same verification for the certificate of $V$ (which might involve contacting his TTP, if $V$ has a different TTP). If the certificates are still valid, then the TTP generates a timestamp TT. Together with the timestamp, the TTP signs the unique certificate identifiers cidU and cidV as well as $g^{s}$. The TTP then sends the timestamp TT, the signature and three cross-certificate chains on $U$ 's, $V$ 's and the TTP's public keys respectively (for the definition of these cross-certificate chains see section 5.2); the parts that may reveal $U$ 's identity are encrypted using $L . V$ forwards the timestamp $T T$, the encrypted signature, and the cross-certificate chain for his public key to $U$, together with the authentication and charging-related parameters presented in the protocol in Figure 1. $U$ can verify the freshness of the signature by the TTP even if he does not have a reliable clock, since the signature also includes $g^{s}$. It is assumed that the user knows the unique identifier number cidU of the certificate on his own public key and that he can derive the identifier cidV from $V$ 's certificate, so that he can verify the received signature. If the signature is correct and the authentication parameters are verified (as for the protocol in Figure 2), the user sends the final message to $V$, which also includes the key $L$ encrypted under the key $K$. Using the key $L, V$ can decrypt the answer received from the TTP in the third step and verify the signature. It is assumed that both TTP and $V$ have a reliable clock, so he can verify TT (it would in fact suffice that the clocks of TTP and $V$ are adequately synchronised).

Next two observations are made on the protocol and its relation to the other protocols in this 
paper:

- Sequence of steps in protocol execution. This protocol has the property that $V$ can only verify the signature of the TTP after the last step, which results in some additional delay. If this is not acceptable, the user can add in the first message a MAC computed with a key derived from $L$ on $i d U$ and $i d V$ (as an opponent will only see a single (text, $M A C$ ) pair for a given key, the required MAC property is weak - but slightly stronger than the weak MAC property discussed in section 2.3). In that case the encryption in the fourth message can use the key $K$ and there is no need to send $L$ in the fifth message. However, this latter solution does not provide protection on the interface between $V$ and TTP.

- Protocol interaction attacks. In this paper, three protocols are proposed in sections 2, 4 and 5 which have similar messages. Therefore, the question arises whether protocol interaction attacks by using (part of) a message from one protocol run in a run of one of the other protocols become possible if the same long-term keys are used in these three protocols. It should be noted first that the final message in any of the three protocols could not be used in any of the other two protocols because the structures of the inputs to the signature are pairwise different. In the re-initialisation protocol of section 4, the final message is the only one of cryptographic relevance. Hence, there is no scope for interaction of this protocol with the other two. Regarding the two authentication protocols with and without on-line TTP in sections 2 and 5 respectively, an inspection shows that an attacker could indeed modify the first two messages exchanged between a user $U$ and a VASP $V$ in such a way that the user believes he was running the protocol without on-line TTP with $V$ whereas in fact $V$ generated the response to $U$ as part of a run of the protocol with on-line TTP. But no harm is seen in this as $U$ gets no incorrect assurances and the protocol will ultimately fail because $V$, expecting a different signature, will reject $U$ 's last message. No further scope for protocol interaction attacks is seen.

\subsection{Cross-certificates and TTP scenarios}

The protocol in section 5.1 uses cross-certificate chains. Such chains are required when parties in the protocol do not have the same TTP, or when the parties do not have on-line access to their TTP. Here $\operatorname{CertChain}(X, Y)$ consists of a sequence of certificates, $c_{0}, c_{1}, \ldots, c_{n}$, where the signer of certificate $c_{0}$ is the Certification Authority (CA) of entity $X$, the subject of $c_{i}$ is equal to the signer of $c_{i+1}(0 \leq i<n)$, and the subject of certificate $c_{n}$ is entity $Y$. Such a certificate is verified starting with $c_{0}$ (using the public key of the CA of entity $X$ ); this guarantees the public key required to verify $c_{1}$, etc. The verification is completed after verification of $c_{n}$. In order to speed up the verification process and reduce the communication overhead, the CA of entity $X$ might also verify the complete chain, and then create a new certificate for entity $Y$. However, this provides slightly different guarantees to the entity verifying the cross-certificate.

For the protocol of section 5.1, a CA structure where the CA of a user $U$ (the TTP) and the CA of a VASP $V$ always cross-certify one another, will provide short certificate chains. Under this assumption (which is not necessary for the correct functioning of the protocol) one has:

- CertChain $(U, V)$ consists of a cross-certificate signed by the user's TTP, and certV.

- CertChain $(V, U)$ consists of a cross-certificate signed by $V$ 's CA, and cert $U$.

- CertChain $(V, T)$ consists of a cross-certificate signed by $V$ 's CA, and a certificate on the signature key used in $\mathrm{Sig}_{T}$ by the user's TTP.

Of course the protocol can be simplified if $U$ and $V$ have the same TTP. Moreover, one can also consider the case where $V$ contacts his own TTP, rather than that of the user. In that case the user should send its certificate $\operatorname{cert} U$ over the air interface in the first protocol step, reducing the efficiency of the protocol and compromising user identity confidentiality. 


\section{Choices for cryptographic algorithms and certificate for- mats}

The protocol and cryptographic mechanisms were chosen in such a way that they are particularly suited to the low bandwidth and low computational capabilities on the user's smart card. The payment protocol itself is very lightweight; the elementary payment operation does not require any public-key operation. The cross-certification approach is chosen in order to minimise communication overheads.

\subsection{Cryptographic algorithms}

The use of elliptic-curve cryptography allows for much shorter signatures and keys; some additional storage is required for the system parameters, but this can be minimised by selecting common system parameters. Current estimates by Wiener [46] indicate that elliptic curves with a 170-bit subgroup order (which typically corresponds to an elliptic curve over a group with $171 \ldots 180$ bits) offer a security level comparable to 1024-bit RSA. The signature scheme used is the AMV scheme of the ISO/IEC CD 15946-2 [28], but the construction of the RSA based signature scheme of ISO/IEC 9796-2 [23] has also been planned as an option for the signature on certificates; recently Coron et al. have pointed out that the security of this standard is smaller than anticipated [8], but for this environment the impact of this attack is very small. The hash function RIPEMD-128 can be used for $h 1$ and $h 2$ in the authentication protocol; this hash function offers a performance in between MD5 (the security of which is questionable, see the results by den Boer and Bosselaers [9] and Dobbertin [11]) and SHA-1 and RIPEMD-160. For collision resistance, 128 bits is on the low side, but for the specific needs of $h 1$ and $h 2$ in the authentication protocol it certainly provides a high security level. For $h 3$ RIPEMD-160 has been selected. For the tick payment protocol, RIPEMD-160 has been selected, restricted to an output of 64 bits.

\subsection{Certificate format}

The advantage of using standard formats for certificates is quite obvious. However, for a specific application, standardised certificates are sometimes much more complex than required. If the application is set in an environment with serious resource constraints regarding storage (e.g., smart cards) or bandwidth (e.g., radio interface, smart card interface) and if the application scenario only requires interactions of participants within a closed (albeit possibly quite large) group, it may be worthwhile thinking about proprietary formats optimised for this particular environment. Proprietary certificate formats are being used in the financial sector (e.g., for the EMV specifications by Europay, Mastercard and VISA). Proprietary certificate formats have also been proposed as an option (in addition to X.509 [30]) for use with mobile data applications by the WAP (Wireless Application Protocol) Forum [45] and in ANSI X9.68 [3].

A special certificate format has been designed for use with the payment application presented in this paper that minimises the storage space on the smart card and the bandwidth on the air interface. The certificate allows for all necessary information: version number, serial number, issuer identifier, four validity dates (begin and end of validity and two optional dates for usage of the private key), subject identifier and public key information (algorithm type identifier and a public key value). Other optional fields include key usage, cross certificate attributes and certificate path attributes. The size of a public-key certificate is less than 200 bytes, which should be compared to about 1 Kbyte for typical X.509 v.1 certificates. The certificate format proposed by WAP was not yet published when the certificate format for the application presented in this paper was specified and implemented. Both proposals aim to reduce the certificate size by restricting the fields in the certificate to the subset needed, avoiding ASN.1 encoding and allowing the use of signature algorithms which yield shorter signatures. They differ in the precise fields and the signature algorithms supported. 


\section{Related work}

There is a vast literature on authentication and key agreement protocols. For overviews, the reader is referred to [36] or [42].

Over the past few years, a substantial number of public key based authentication and key agreement protocols have also been proposed specifically for a mobile environment. For an overview and evaluation of these protocols see Horn et al. [22]. Only a few are mentioned here.

A well-established authentication and key agreement protocol is the Station-to-Station (STS) protocol with its variants [10]. It meets all the goals listed in section 2.1. (Initiation of payment could also be easily integrated with the STS protocol.) The STS protocol was not specifically designed for a mobile environment. Compared with the protocol presented here it has somewhat longer messages and higher computational requirements, irrespective of the choice of the signature and key agreement mechanisms.

A quite efficient public key based protocol designed for a mobile environment which was published recently is the Boyd-Park protocol [7]. It can be easily modified so that it appears to satisfy all the protocol goals in section 2.1. With the appropriate choice of the cryptographic algorithms, it is computationally more efficient on the user side than the protocol presented here, at the expense of longer messages and an additional initial pass to distribute the VASP's certificate, making it essentially a four pass protocol.

Further public key based protocols for a mobile environment were proposed in $[4,5,34,38,39$, $47,48]$. None of these protocols, however, meets all the goals listed in section 2.1.

\section{Conclusion}

The protocols presented in this paper provide an efficient way to achieve mutual authentication, key establishment and incontestable charging in a mobile environment. The protocol satisfies the needs for UMTS: it requires a low computational load on the user's side and requires only a limited amount of communication. Moreover, it can be extended to a large scale system with multiple TTPs through cross-certification.

\section{References}

[1] Information on 3GPP available from www.3gpp.org.

[2] R. Anderson, H. Manifavas, C. Sutherland, A practical electronic cash system, available from www.cl.cam.ac.uk/users/rja14/.

[3] ANSI X9.68, Digital Certificates for Mobile, Account Based, and High Transaction Volume Financial Systems, ASC X9 Secretariat, American Bankers Association, draft, January 1998.

[4] A. Aziz, W. Diffie, Privacy and Authentication for Wireless Local Area Networks, IEEE Personal Communications, 1st Q 1994, 25-31.

[5] M.J. Beller, Y. Yacobi, Authentication and key agreement protocol for PCS, Joint experts meeting on privacy and authentication for PCS, P\&A JEM/93-012, Nov. 8, 1993.

[6] J. Borst, B. Preneel, J. Vandewalle, On the time-memory tradeoff between exhaustive key search and table precomputation, in: Proc. 19th Symposium on Information Theory in the Benelux, Veldhoven (NL), 1998, pp. 111-118.

[7] C. Boyd, D.-G. Park, Public key protocols for wireless communications, in: Proc. of The 1st International Conference on Information Security and Cryptology (ICISC'98), December 18-19, 1998, Seoul, Korea, pp. 47-57.

[8] J.-S. Coron, D. Naccache, J.P. Stern, On the security of RSA padding, in: Proc. Crypto'99, Lecture Notes in Computer Science 1666, M. Wiener, ed., Springer-Verlag, 1999, pp. 1-18. 
[9] B. den Boer, A. Bosselaers, Collisions for the compression function of MD5, in: Proc. Eurocrypt'93, Lecture Notes in Computer Science 765, T. Helleseth, ed., Springer-Verlag, 1994, pp. 293-304.

[10] W. Diffie, P. van Oorschot, M. Wiener, Authentication and authenticated key exchanges, Designs, Codes and Cryptography 2 (1992), 107-125.

[11] H. Dobbertin, The status of MD5 after a recent attack, CryptoBytes 2 (2)(1996), 1-6.

[12] H. Dobbertin, A. Bosselaers, B. Preneel, RIPEMD-160: a strengthened version of RIPEMD, in Fast Software Encryption, Lecture Notes in Computer Science 1039, D. Gollmann, ed., Springer-Verlag, 1996, pp. 71-82. See also www. esat.kuleuven. ac.be/ bosselae/ripemd160.

[13] T. ElGamal, A public key cryptosystem and a signature scheme based on discrete logarithms, IEEE Trans. on Information Theory IT-31 (4) (1985), 469-472.

[14] Information on ETSI available from www.etsi.fr.

[15] ETSI ETS GSM 02.09, European Digital Cellular Telecommunications System (Phase 1); Security Aspects, Version 3.1.0, European Telecommunications Standards Institute, 1993.

[16] ETSI ETS GSM 03.20, European Digital Cellular Telecommunications System (Phase 1); Security-related Network Functions, Version 3.3.2, European Telecommunications Standards Institute, 1992.

[17] ETSI ETS 300175-7, DECT Common Interface, Part 7: Security Features, European Telecommunications Standards Institute, 1992.

[18] FIPS 180-1, Secure Hash Standard, Federal Information Processing Standard (FIPS), Publication 180-1, National Institute of Standards and Technology, US Department of Commerce, Washington D.C., 1995.

[19] The GSM Association: www.gsmworld.com.

[20] R. Hauser, M. Steiner, M. Waidner, Micro-payments based on iKP, Presented at SECURICOM 96. Available from www.zurich.ibm.com.

[21] M. Hellman, A cryptanalytic time-memory tradeoff, IEEE Trans. Information Theory $\mathbf{I T - 2 6}$ (1998), 401-406.

[22] G. Horn, K.M. Martin, C.J. Mitchell, Authentication protocols for mobile network environment value-added services. Unpublished, Technical Report, ESAT-COSIC, Katholieke Universiteit Leuven (B), 1999.

[23] ISO/IEC 9796-2, Information technology - Security techniques - Digital signature schemes giving message recovery, Part 2: Mechanisms using a hash-function, International Organization for Standardization, 1997.

[24] ISO/IEC 10118, Information technology - Security techniques - Hash-functions, Part 1: General, International Organization for Standardization, 1994, Part 2: Hash-functions using an n-bit block cipher algorithm, International Organization for Standardization, 1994, Part 3: Dedicated hash-functions, International Organization for Standardization, 1998.

[25] ISO/IEC 11770-3, Information technology - Security techniques - Key management, Part 3: Mechanisms using asymmetric techniques, International Organization for Standardization, 1997.

[26] ISO/IEC 13888-3, Information technology - Security techniques - Non-repudiation, Part 1: General model, International Organization for Standardization, 1997. 
[27] ISO/IEC 14888-3, Information technology - Security techniques - Digital signature with appendix, Part 1: General, Part 3: Certificate-based mechanisms, International Organization for Standardization, 1999.

[28] ISO/IEC CD 15946-2, Information technology - Security techniques - Cryptographic techniques based on elliptic curves, International Organization for Standardization, Committee Draft, 1999.

[29] Information on ITU available from www.itu.int.

[30] ITU-T Recommendations X.509, Authentication Framework, Geneva 1989.

[31] C.S. Jutla, M. Yung, Paytree: Amortised-signature for flexible micropayments, in: Proc. of Second USENIX Association Workshop on Electronic Commerce, November 1996, pp. 213221.

[32] L.R. Knudsen, K. Martin, B. Preneel, One-way functions for tick payments. Unpublished, Technical Report, ESAT-COSIC, Katholieke Universiteit Leuven (B), 1999.

[33] L. Lamport, Password authentication with insecure communication, Communications of the ACM 24 (1981), 770-772.

[34] H. Lin, L. Harn, Authentication in wireless communications, in: Proc. GLOBECOM 1993, IEEE, 1993, pp. 550-553.

[35] A. Menezes, Elliptic Curve Public Key Cryptosystems, Kluwer Academic Publishers, Boston, 1993.

[36] A. Menezes, P. van Oorschot, S. Vanstone, Handbook of Applied Cryptography, CRC Press, Boca Raton, 1997.

[37] C.J. Mitchell, M. Ward, P. Wilson, Key control in key agreement protocols, Electronics Letters 43 (1998), 980-981.

[38] Y. Mu, V. Varadharajan, On the design of security protocols for mobile communications, in: Information security and privacy, Lecture Notes in Computer Science 1172, J. Pieprzyk, J. Seberry, eds., Springer-Verlag, 1996, pp. 134-145.

[39] C.-S. Park, On certificate-based security protocols for wireless mobile communication systems, IEEE Network 11 (5) (1997), 50-55.

[40] T. Pedersen, Electronic payments of small amounts, in: Security Protocols, Lecture Notes in Computer Science 1361, M. Lomas, ed., Springer-Verlag, 1997, pp. 59-68. See also DAIMI PB-495, Technical Report, Computer Science Department, Aarhus University, August 1995.

[41] R.L. Rivest, A. Shamir, PayWord and MicroMint: Two simple micropayment schemes, in: Security Protocols, Lecture Notes in Computer Science 1361, M. Lomas, ed., Springer-Verlag, 1997, pp. 69-87. Extended version also available from http://theory.lcs.mit.edu/ rivest

[42] R. Rueppel, P. van Oorschot, Modern key agreement techniques, Computer Communications 17 (7) (July 1994), 458-465.

[43] UMTS Forum, reports nos. 1-6, available from http://umts-forum.org/.

[44] K. Vedder, Security aspects of mobile communications, in: Computer Security and Industrial Cryptography. State of the Art and Evolution, Lecture Notes in Computer Science 741, B. Preneel, R. Govaerts, J. Vandewalle, eds., Springer-Verlag, 1993, pp. 193-210. 
[45] WAP Forum, Wireless Application Protocol, Wireless Transport Layer Security Specification, version 30-Apr-1998, available from www.wapforum.com.

[46] M. Wiener, Performance comparisons of public-key cryptosystems, CryptoBytes 4 (1)(1998), $1-5$.

[47] X. Yi, E. Okamoto, K.-Y. Lam, An optimized protocol for mobile network authentication and security, Mobile Computing and Communications Review 2 (3) (1998), 37-39.

[48] J. Zhou, K.-Y. Lam, Undeniable billing in mobile communications, in: Proc. of the 4th ACM/IEEE International Conference on Mobile Computing and Networking, ACM Press, Dallas (Texas), 1998.

\section{Acknowledgements}

The authors would like to thank the following colleagues for their help and advice: Volker Kessler and Klaus Müller contributed to the development of the protocol presented in section 2. Peter Howard, Lars Knudsen, Keith Martin, Chris Mitchell and Konstantinos Rantos provided valuable technical input and useful comments. We would also like to thank all the partners in the ASPeCT project for their good cooperation, and the anonymous referees for their helpful comments.

The second author, Bart Preneel is a research associate sponsored by the Fund for Scientific Research, Flanders (Belgium). 
USER $U$

VASP $V$

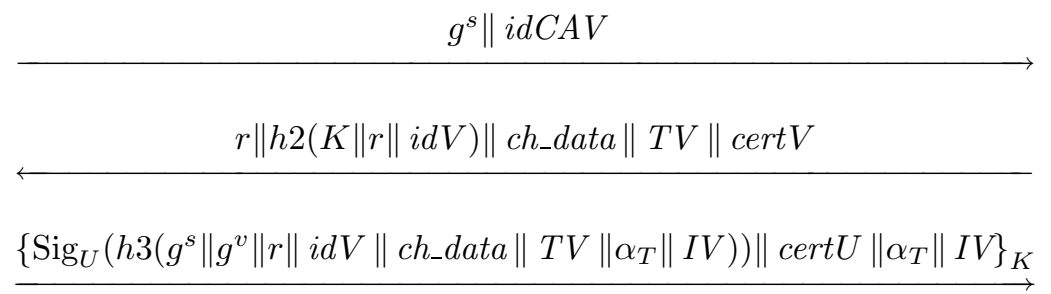

Figure 1: The authentication and initialisation of payment protocol. 


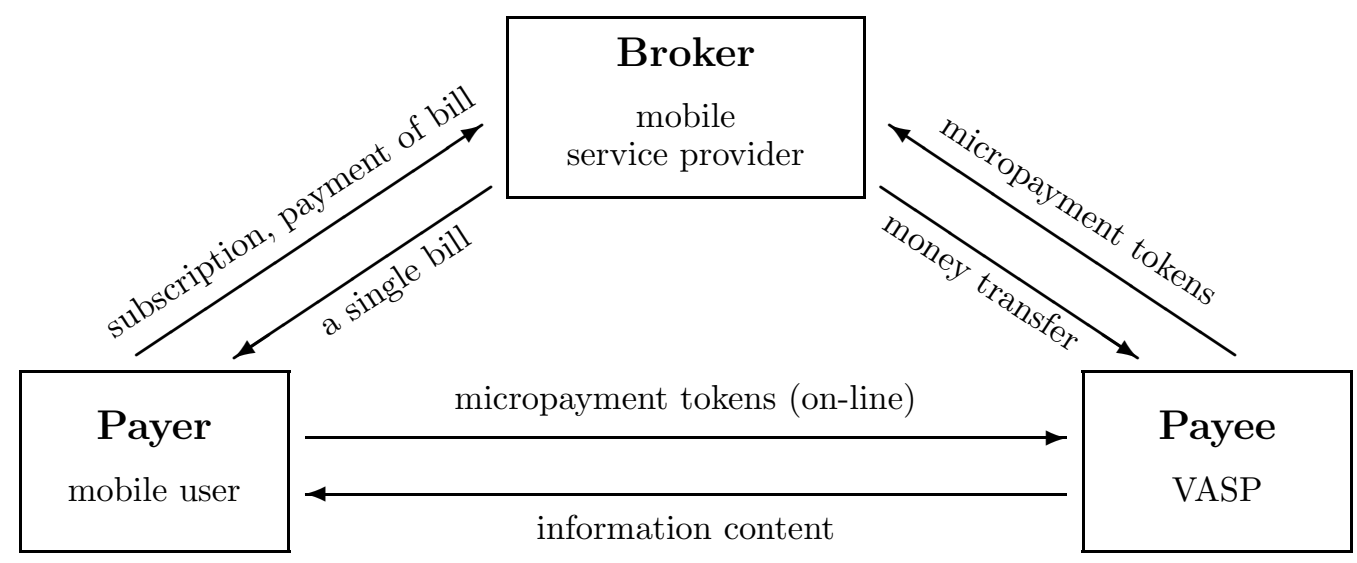

Figure 2: Model of the payment system. 


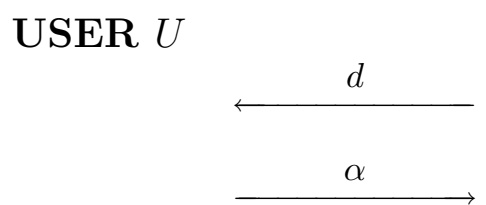

VASP $V$

Figure 3: The tick payment protocol. 
USER $U$

VASP $V$

ch_data $\| T V$

$\left\{\operatorname{Sig}_{U}\left(h 3\left(r\|i d V\| \text { ch_data }\|T V\| \alpha_{T} \| I V\right)\right)\left\|\alpha_{T}\right\| I V\right\}_{K}$

Figure 4: The re-initialisation protocol. 
$g^{s}\|i d T T P\|\{i d U\}_{L}$

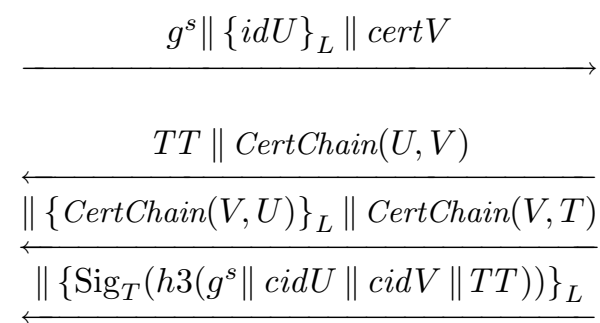

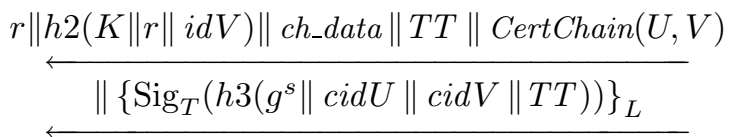

$\left\{\operatorname{Sig}_{U}\left(h 3\left(g^{s}\left\|g^{v}\right\| r\|i d V\|\right.\right.\right.$ ch_data $\left.\left.\|T T\| \alpha_{T} \| I V\right)\right)$

$\left.\left\|\alpha_{T}\right\| I V \| L\right\}_{K}$

Figure 5: The authentication and key agreement protocol with on-line TTP. 